\title{
Effects of noise and detection error in a dynamic adaptive optics system
}

\author{
She Chen, Hai-Xing Yan*, Shu-Shan Li \\ (Institute of Mechanics, Chinese Academy of Sciences, Beijing 100080, China)
}

\begin{abstract}
It is well known that noise and detection error can affect the performances of an adaptive optics (AO) system. Effects of noise and detection error on the phase compensation effectiveness in a dynamic AO system are investigated by means of a pure numerical simulation in this paper. A theoretical model for numerically simulating effects of noise and detection error in a static AO system and a corresponding computer program were presented in a previous article. A numerical simulation of effects of noise and detection error is combined with our previous numeral simulation of a dynamic AO system in this paper and a corresponding computer program has been compiled. Effects of detection error, readout noise and photon noise are included and investigated by a numerical simulation for finding the preferred working conditions and the best performances in a practical dynamic AO system. An approximate model is presented as well. Under many practical conditions such approximate model is a good alternative to the more accurate one. A simple algorithm which can be used for reducing the effect of noise is presented as well. When signal to noise ratio is very low, such method can be used to improve the performances of a dynamic AO system.
\end{abstract}

KEY WORDS: Dynamic adaptive optics system, Numerical simulation, Noise, Detection error, Approximate model, Algorithm.

\section{INTRODUCTION}

It is well known that an adaptive optics (AO) system can realize the real-time wavefront detection and correction to significantly improve the image quality of optical wave after propagating in a turbulent atmosphere. Therefore, it has been widely applied in improving the astronomical observation of ground-based telescope and the laser beam propagation. $^{1,2}$

Because an AO system and its working condition are not ideal, its performances have been limited. ${ }^{3-5}$ There are three important factors to affect the performances in an AO system. The first of them is the limited spatial bandwidth resulted from the limited numbers of the sub-apertures for wavefront detection and the actuators in the deformable mirror for wavefront correction. The second is the limited temporal bandwidth resulted from the limited response speed for wavefront detection, reconstruction and correction. This is the main subject of a dynamic AO system. ${ }^{6}$ The third is effects of noise and detection error. ${ }^{7,8}$ This paper will focus on effects of the third factor in a dynamic AO system.

* Correspondence: Email: hxyan@imech.ac.cn; Phone: (86) 10 62554123; Fax: (86) 1062561284. 
The effects of noise and detection error on performances of an AO system are in terms of the measurement error of optical centroid in the widely-used Hartmann-Shack (HS) wavefront sensor. The effects of noise and detection error in an $\mathrm{AO}$ system can be divided into three parts: ${ }^{9,10}$ (i) detection error from the number-limited discrete sampling (herein, there are two kinds of limited numbers, first, the limited number of subapertures in a HS sensor, second, the limited number of CCD pixels for detecting the optical centroid of each subaperture, the first one was discussed in other papers $^{11,12}$ and only the second one is included in this paper); (ii) readout noise; and (iii) photon noise. The effects of noise and detection error in a static AO system were investigated in Refs.[7,8]. Based on a similar idea, the effects of noise and detection error in a dynamic $\mathrm{AO}$ system are investigated in this paper.

Firstly, these three parts are theoretically analyzed and an analytical formula describing effects of readout noise and photon noise on a HS sensor is derived. Based on the theoretical analysis, a theoretical model for numerically simulating the effects of noise and detection error in an AO system is presented. A corresponding computer program is compiled and combined with our existing computation program of numerical simulation of laser propagation in the atmosphere and a dynamic AO system. A series of computational results are obtained to do numerical simulation investigations. These results are very useful for the design and application of a practical AO system.

In this paper, an algorithm which can be used for decreasing the noise effect in an AO system is presented and preliminary computational results are presented as well.

\section{THEORETICAL ANALYSIS}

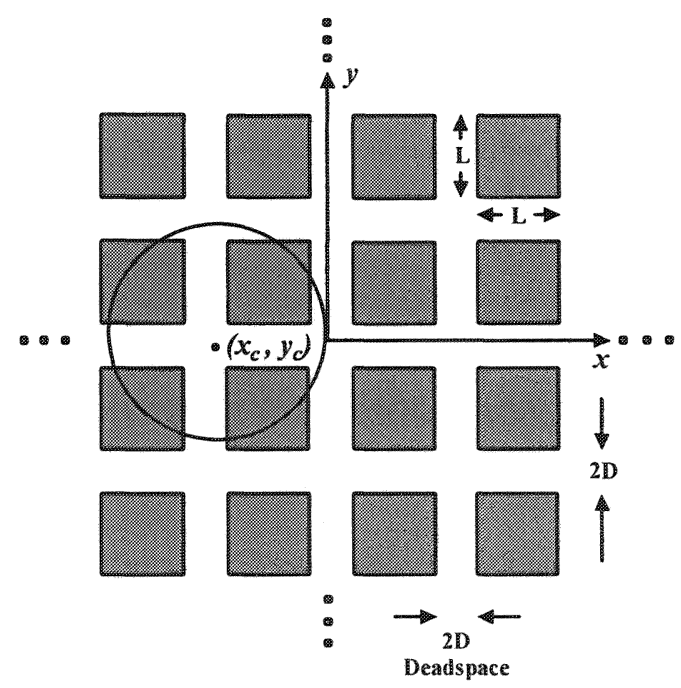

Fig. 1 Diagram of detecting the optical centroid by means of a discrete CCD array.

There are two reasons to create detection error in a HS wavefront sensor. Firstly, number-limited discrete CCD pixels are used to measure the optical centroid for each subaperture. Secondly, there is a deadspace between two adjacent CCD pixels. In fact, there is a third reason, i.e. difference and interference among pixels (see Fig.1). The last one has not been 
included in this paper, because lacking enough experimental data that can be used. It is difficult for the detection error to derive an accurate analytical formula to describe its effect on determining centroid in a HS sensor. However, it is natural and convenient in a numerical simulation to numerically calculate focusing and centroiding of turbulence-distorted optical wave for each subaperture according to the realistic distribution of CCD pixels for that subaperture. In more details, the wave field of the beam that radiates from a beacon propagates through a turbulent atmosphere and strikes the HS sensor. Then, the wave field is divided into several subapertures and the wave field of a subaperture propagates to reach the focal plane (CCD pixels) of the HS sensor. The centroid position for each subaperture can be calculated by using the detected photon events in all related CCD pixels. Furthermore, the average slopes of the subaperture in $x$ and $y$ directions can be determined. It is completely natural to include the effects of the detection error on a HS wavefront sensor in a numerical simulation.

Effects of the readout noise and the photon noise on a HS sensor are in terms of affecting the photon events detected by each CCD pixel. ${ }^{9,13}$ A centroid position of an image spot detected by a group of CCD pixels is given by the following expressions for $x_{c}$ and $y_{c}$

$$
x_{c}=\sum_{i, j}^{N} x_{i} n_{i j} / n_{i j}, \quad y_{c}=\sum_{i, j}^{N} y_{j} n_{i j} / n_{i j}
$$

where $x_{i}, y_{j}$, and $n_{i j}$ are the coordinates and photon events associated with the pixel in column $i$ and row $j$ of the CCD array, respectively, $N$ is the total pixel number for each subaperture. When the readout noise and the photon noise are included, the variance of $n_{i j}$ is

$$
\sigma_{i j}^{2}=\sigma_{p}^{2}+\sigma_{r}^{2}
$$

where $\sigma_{r}{ }^{2}$ is the variance of the readout noise and $\sigma_{p}{ }^{2}$ is the variance of the photon noise. The photon noise obeys a Poisson statistics and the variance in a Poisson statistics is equal to the average. That is, $\sigma_{p}{ }^{2}=n_{i j}$. According to the generalized law of error propagation, the error variance $\sigma_{x_{c}}^{2}$ in $x_{c}$ can be derived as

$$
\sigma_{x_{c}}^{2}=\left(r_{x}^{2}+\sigma_{r}^{2} f\right) / \sum_{i, j}^{N} n_{i j}
$$

where

$$
\begin{gathered}
r_{x}=\left(\overline{x^{2}}+\bar{x}^{2}\right)^{1 / 2}, \\
f=\left(\sum_{i, j}^{N} x_{i}^{2}+N \bar{x}^{2}-2 \bar{x} \sum_{i, j}^{N} x_{i}\right) / \sum_{i, j}^{N} n_{i j} \\
\bar{x}=\left(\sum_{i, j}^{N} x_{i} n_{i j}\right) / \sum_{i, j}^{N} n_{i j}
\end{gathered}
$$




$$
\overline{x^{2}}=\left(\sum_{i, j}^{N} x_{i}^{2} n_{i j}\right) / \sum_{i, j}^{N} n_{i j} .
$$

Eq. (3) is the analytical expression of effects of the readout noise and the photon noise on the $x_{c}$ for a subaperture. The expression for the error in $y_{c}$ is identical except that $y_{j}$ will substitute for $x_{i}$.

\section{NUMERICAL COMPUTATION}

When numerically calculating effects of the readout noise and the photon noise on an AO system, two methods have been utilized. The first of them is called as formulation method. In detail, Eq. (3) is used to calculate the variance $\sigma_{x_{c}}^{2}$ in $x_{c}$ for a subaperture in the formulation method, then, a random process which variance is equal to $\sigma_{x_{c}}^{2}$ is assumed to obey the Gauss statistics to randomly produce the change (error) of $x_{c}$ due to effects of the readout noise and the photon noise for that subaperture in a random process realization. This change (error) of $x_{c}$ is then added to the $x_{c}$ without any noise to obtain the centroid position in $x$ direction with noise effects. The centroid position in $y$ direction with noise effects can be calculated in the same way. It is shown in the later computational results that the analytical formula is a very good approximation on the condition of higher signal-to-noise ratio (SNR); but, when SNR is smaller, results of the formulation method differ from the practical results. It may be because some approximations are included in deriving Eq. (3) and a Gauss statistics is assumed for the random process producing error in the centroid position. Another factor may be more important, that is, the density of photon is not uniform over the focal plane. It is neglected in the formulation method. The analytical formula Eq. (3) may be useful in system analysis and error estimation.

The second method is called as statistics method. In this method, effects of the readout noise and the photon noise on an AO system are directly computed in a numerical simulation. In detail, it is considered that the photon events $n_{i j}$ in Eq. (1) must add a random increment $\Delta n_{i j}$ due to the photon noise having variance $n_{i j}$, which obeys a Poisson statistics, and due to the readout noise having variance $\sigma_{r}{ }^{2}$, which is assumed to obey a Gauss statistics. The random increment $\Delta n_{i j}$ is added to $n_{i j}$ without any noise to be substituted into Eq. (1) to directly obtain the centroid position with noise effects. In this way, effects of the readout noise and the photon noise on an AO system are accurately and naturally included in the calculated slopes.

Next, a numerical simulation of effects of the readout noise and the photon noise on an AO system is combined with our existing numerical simulation of laser beam propagation in the turbulent atmosphere and a dynamic $\mathrm{AO}$ system ${ }^{6,11}$ to do numerical simulation investigations of the effects of readout noise and photon noise on the performances of a dynamic AO system. A number of patterns at target corresponding to different dynamic iterations are accumulated to obtain a long-exposure pattern. In this paper, a number of 1024 for the dynamic iterations is used. A centroid is determined from the long-exposure pattern. The Strehl ratio $S$ is defined as the ratio of the optical energies within a circle around the centroid with a radius of the first dark ring in the Airy pattern after propagations through the turbulent medium and through a vacuum. $S$ in this paper is STRCC in Ref.11, and is used to evaluate the phase compensation effectiveness of an AO system. The whole process is similar to the experimental observation.

In the next section, taking the long-exposure Strehl ratio $S$ as an evaluation parameter, numerical simulation results of 
effects of the noises and the detection error on a dynamic AO system are presented. It is the long-exposure Strehl ratio $S$ which is mostly concerned by experimenters and is most widely used in the experimental observation. Although there are many factors to affect $S$ in quite complicated ways, most results presented in the next section relate to the effect of an individual factor. Therefore, it is possible to evaluate the effect of an individual factor on the phase compensation effectiveness by using the effect of that factor on $S$.

As a random variable, noise obeys the same statistics law of such variable. As well known, if you want to reduce the variance of a random variable, it is the easiest way to summarize the variable several times and to obtain an average of the summation. By such a way, a better result with less variance can be gotten. The resultant variance is

$$
\sigma_{r}^{2}=\sigma_{r}^{2} / n
$$

where $\sigma_{r}^{2}$ is the original variance, $\sigma_{r}{ }^{2}$ is the resultant variance, $n$ is the times number that the original variable has been summarized. In a numerical simulation, in order to realize this idea it is needed to get the summation of several centroids with the effect of noises (in this paper, the number is taken as 4) and then to get an average of the summation. Using such average to reconstruct the distorted wavefront, the effect of noises can be reduced. Because several distorted wavefronts have been used to reconstruct one wavefront, the delay in an AO system should be prolonged. If we want to utilize such idea to get some benefits, there are two limitations: firstly, the turbulent change in the atmosphere is not very fast, and the detection frequency is fast enough; secondly, the performance of a dynamic AO system is greatly decreased by the noises. In the next section, this idea is tested under such conditions.

\section{RESULTS AND DISCUSSIONS}

In this section, first, the effect of the size of focal spot in a subaperture on the computational results is shown and discussed. Because effects of different factors in the noises and the detection error on an AO system have some cross-linking, for easily understanding, results and discussions are divided into three portions: effect of the number-limited discrete sampling error; effect of the readout noise and the effect of the photon noise; then, result under the condition of detection error, the readout noise and the photon noise existing simultaneously is presented. Further, the statistics method and the formulation method in computing effects of the readout noise and the photon noise are compared. Finally, some preliminary result of the proposed method for reducing noise effect is presented.

\subsection{Effect of spot radius for each subaperture on the computational results}

In the numerical computation, it is found that under the condition of a certain sampling density which is defined as the CCD pixel number for each subaperture, when the spot radius on the focal plane (i.e. on the CCD target plane) of each subaperture is different, i.e. when relative comparison of the spot size to the CCD pixel size is different, the wavefront detection and phase compensation in an AO system are influenced. Computational results for several interesting sampling densities are shown in Fig. 2. The CCD pixel number for each subaperture is shown within the rectangle in Fig. 2 and the computational grid number for each subaperture is $64 \times 64$. It is shown in Fig. 2 that there is an optimum spot radius for each sampling density. Under this optimum condition the phase compensation effectiveness is optimum. This optimum spot radius can be expressed by the computational grid number within the spot radius. It is noted that the effects of the spot radius in the numerical simulation depend on the sampling density and the optimum spot radius is 
different for a different sampling density. The following computational results are obtained on such optimum condition for different sampling density.

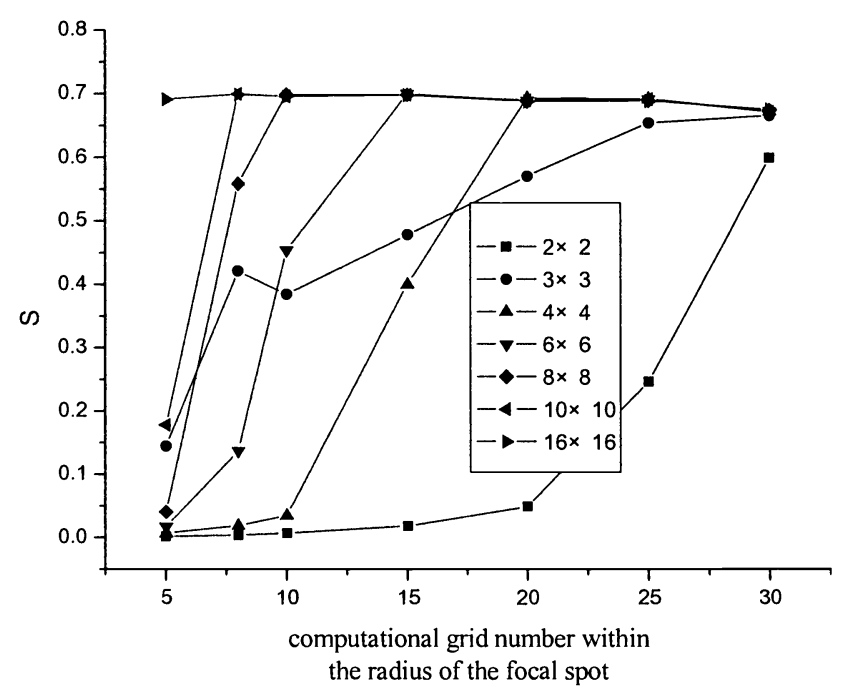

Fig. 2 Effects of the radius of focal spot for each subaperture on the computational results in a numerical simulation.

Computational conditions: horizontal propagation of a laser beam within distance of one kilometer, focused beam, wavelength of both beacon beam and main laser beam is 0.8200 micrometer, 10 phase screens, $C_{n}^{2}=10^{-14.6} \mathrm{~m}^{-2 / 3}$, atmospheric coherence length $r_{0}=7.6104 \mathrm{~cm}$; AO system of 61 elements (with 48 subapertures), there is no deadspace between the CCD pixels, without the readout noise and the photon noise, lateral wind speed $v=1 \mathrm{~m} / \mathrm{s}$, the frame frequency of CCD is $2900 \mathrm{~Hz}, 1024$ dynamic iterations.

\subsection{Error of number-limited discrete sampling}

There are two factors in this aspect: the sampling density expressed by the corresponding CCD pixel number for each subaperture and size of the deadspace between two adjacent CCD pixels.

\subsubsection{Sampling density}

It can be seen from Fig. 3 that when the sampling density is larger than a certain value the computational results are substantially same. This certain value can be thought as the necessary pixel number for the AO system on the corresponding condition. Although a further increase of the sampling density can produce a small amount of performance improvement, this increase will result in a corresponding increase of total CCD pixel number to create a decrease of sampling rate and thus a longer time delay. These negative effects on the performance of an AO system may be larger than the small amount of performance improvement. Therefore, it is quite important to determine this certain value of necessary CCD pixel number. This value has a direct relationship to the practical arrangements in an $A O$ system. 


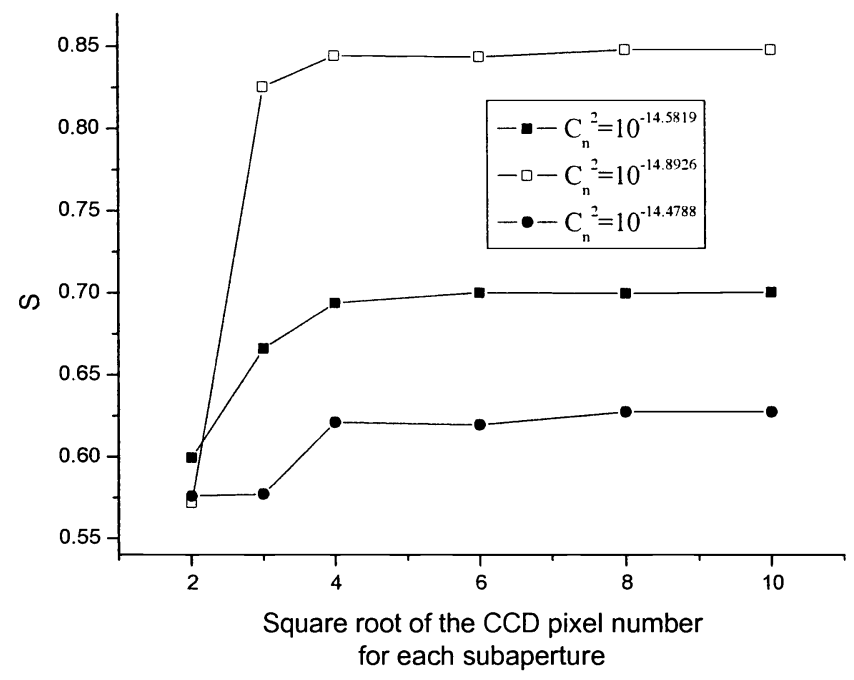

Fig. 3 Effects of sampling density on the computational results of numerical simulation.

Computational conditions: same as those in Fig. 2 besides those indicated in this figure.

\subsubsection{Deadspace between two adjacent CCD pixels}

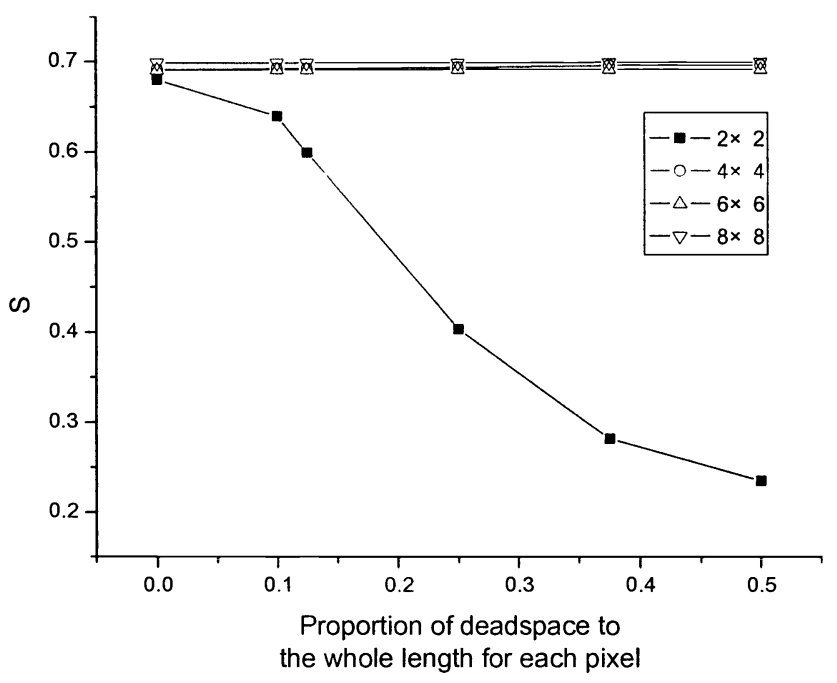

Fig. 4 Effects of the deadspace between two CCD pixels on the computational results of numerical simulation.

Computational conditions: same as those in Fig. 2 besides those indicated in this figure.

It can be seen from Fig. 4 that when the sampling density expressed by the CCD pixel number for each subaperture is different, the effect of the deadspace size on an AO system is also different. The deadspace size is expressed by proportion of the deadspace, which is defined as ratio of deadspace length and total length of each pixel (in Fig. 1 this deadspace proportion is $2 D /(2 D+L))$. In the case of larger pixels number ( $4 \times 4$ and higher), the deadspace almost has no obvious effect on the performance of a dynamic AO system. There is another factor which must be considered, that is, 
as deadspace size increases, the corresponding effective detection area of CCD device decreases and thus detectable photon events decrease to create larger noise. In the case of smaller pixel number (here, $2 \times 2$ ), the effect of deadspace is quite significant. In practice, the deadspace proportion is generally smaller than 0.125 . Our simulation computation shows that on this condition the effect of deadspace is not important for a higher sampling density ( $4 \times 4$ and higher). But when the sampling density is $2 \times 2$, the effect of deadspace is significant.

Besides the two factors mentioned above, there are some other factors in a practical AO system to affect the wavefront detection and further to influence the performance of the AO system, such as nonuniformity in size and/or sensitivity of CCD pixel, failure of individual pixel, nonuniformity in deadspace size, etc. By using a numerical simulation of this paper, all these factors can be conveniently calculated and investigated in a quantitative way.

\subsection{Effect of the readout noise}

It can be seen from Fig. 5 that when the $\mathrm{SNR}\left(\mathrm{SNR}=n_{i j} / \sigma_{r}\right.$ for the readout noise) is larger than a certain value, Strehl ratio $S$ approaches to a stable value. In the computation a typical photon event number is chosen. However, in practice this number is related to the SNR.

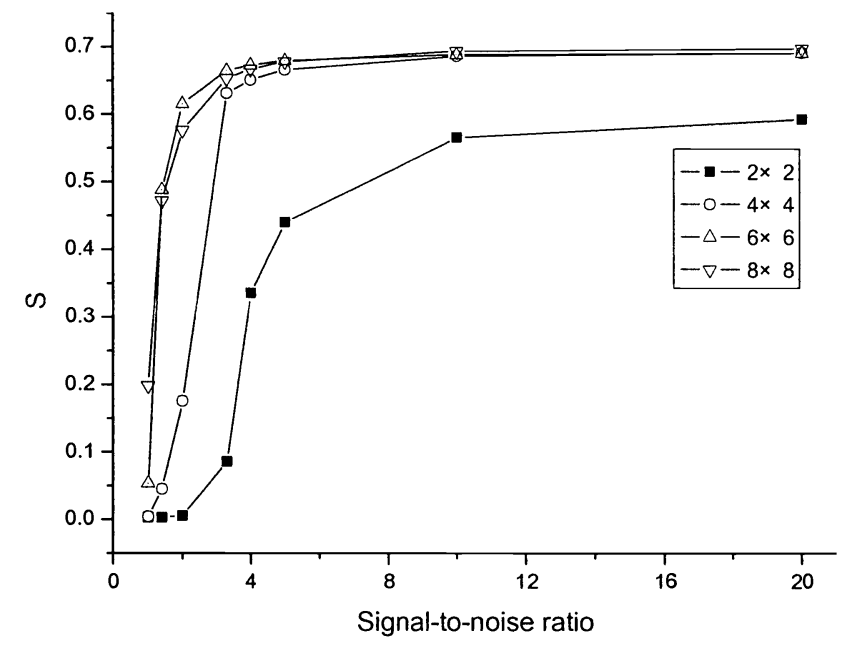

Fig. 5 Effects of the readout noise on the computational results of numerical simulation.

Computational conditions: without the photon noise, proportion of the deadspace is 0.125 , counts of photon events for each CCD pixel are 10, and others are same as those in Fig. 2 besides those indicated in this figure.

\subsection{Effect of the photon noise}

Results shown in Fig. 6 are quite similar to those in Fig. 5. Again, SNR (SNR $=n_{i j} / \sqrt{n_{i j}}$ for the photon noise) is the determining parameter. When SNR is larger than a certain value, Strehl ratio $S$ approaches to a stable value. Note that because the photon noise only relates to the photon events, in order to decrease the effect of photon noise the photon event counts must be larger than a certain value. 


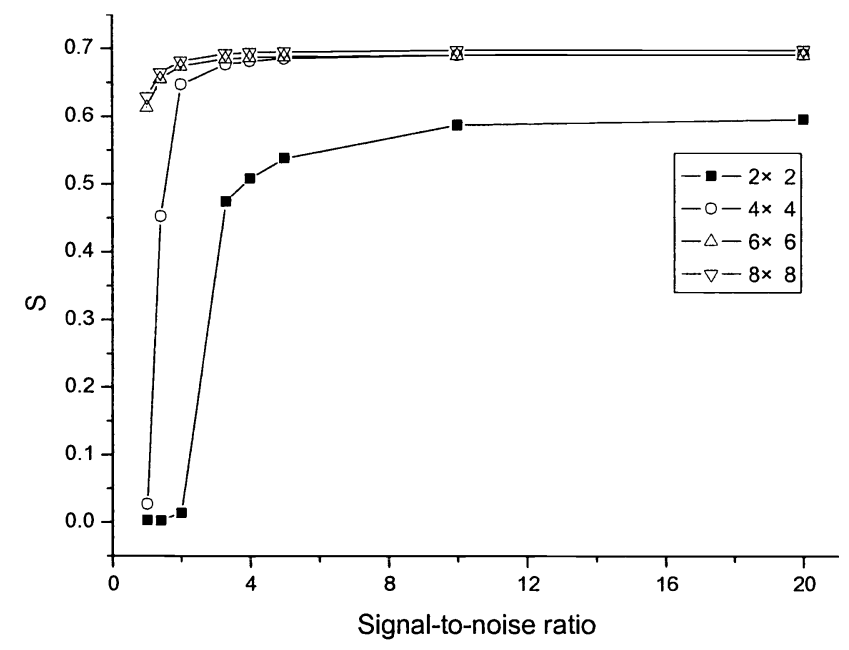

Fig. 6 Effects of the photon noise on the computational results of numerical simulation.

Computational conditions: without the readout noise, proportion of the deadspace is 0.125 ; others are same as those in Fig. 2 besides those indicated in this figure.

\subsection{Comprehensive effect of three factors of noises and detection error}

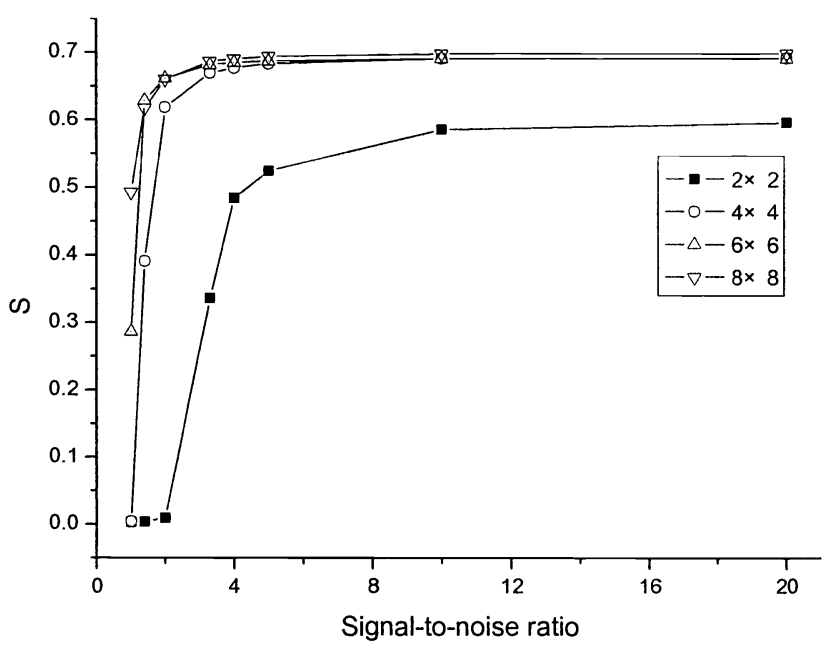

Fig. 7 Effects of the photon noise and the readout noise on the computational results of numerical simulation.

Computational conditions: proportion of the deadspace is $0.125, \sigma_{r}=2$, others are same as those in Fig. 2 besides those indicated in this figure.

Hereinabove the respective effects of the number-limited discrete sampling, the readout noise and the photon noise are discussed. However, in fact these three factors exist in an AO system at the same time and the performance of the AO system is practically influenced by a comprehensive effect of these factors. The effect of SNR on the performance of an AO system when readout noise and photon noise exist at the same time is shown in Fig. 7. It can be seen from Fig. 7 
that in order to obtain a certain phase compensation effectiveness the SNR in an AO system must be large enough; if SNR is too small, the measurement error becomes larger extremely fast; when SNR is larger than a certain value (here, about 5 for a higher sampling density), the improvement of the AO system performance is quite limited.

\subsection{Comparison of the statistics method with the formulation method}

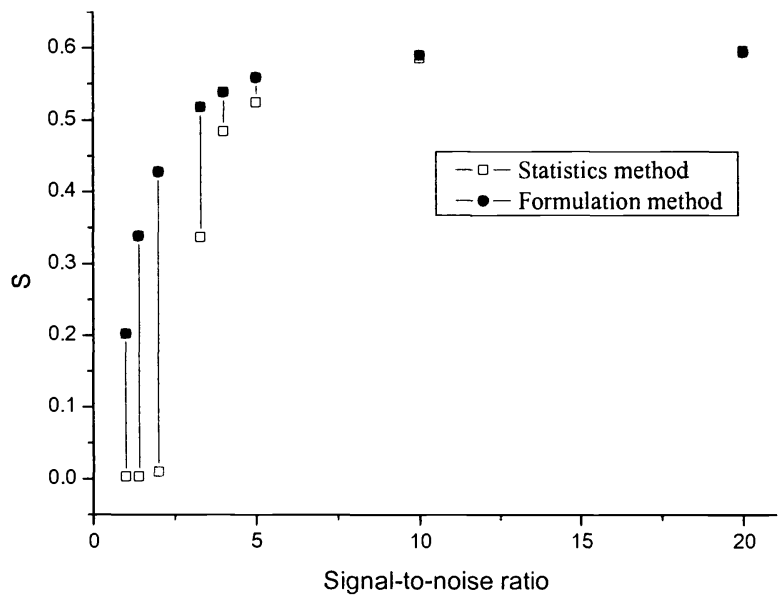

Fig. 8-1 Comprehensive effect of several factors on the computational results of numerical simulation.

(The pixel number for each subaperture is $2 \times 2$ )

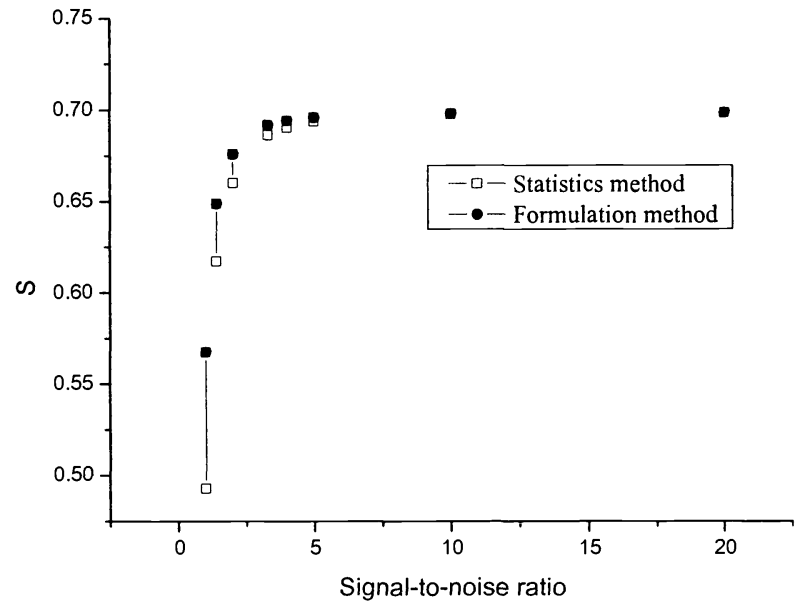

Fig. 8-2 Comprehensive effect of several factors on the computational results of numerical simulation.

(The pixel number for each subaperture is $4 \times 4$ )

Computational conditions: proportion of the deadspace is $0.125, \sigma_{r}=2$, others are same as those in Fig. 2 besides those indicated in this figure.

A comparison of computational results of numerical simulation by using the statistics method with those by using the formulation method is shown in Fig.8. It can be seen from Fig.8 that when SNR is larger, results of the formulation method are almost same as those of the statistics method; but, when SNR is smaller, results of the formulation method are notably different in comparison to those of the statistics method.

\subsection{Preliminary study of the proposed method for reducing the effect of noise.}

It can be seen from Fig. 9 that the effect of noise has been reduced by the method proposed in this paper. Nevertheless, the result is not as good as we expected. This result is not inscrutable. Several detected wavefronts have been summarized to reconstruct one wavefront. At the same time, the sampling time has been prolonged. Our understanding at present time is as follows: in fact, these two processes compete with each other in this method. Summarizing and averaging several wavefronts with effect of random noise can reduce the noise effect. This process is favorable for improving the performance of an AO system. Another process is to prolong the sampling time period i.e. to decrease the sampling frequency. This is unfavorable for the performance in a dynamic AO system. The final benefit depends on the competition of these two processes in the AO system. When the pixel number for each subaperture is lower such as $2 \times 2$, the noise effect may be greater than that of higher sampling density and this method for reducing the noise effect seems 
more effective for improving the performance of a dynamic AO system under the condition of higher frame frequency.

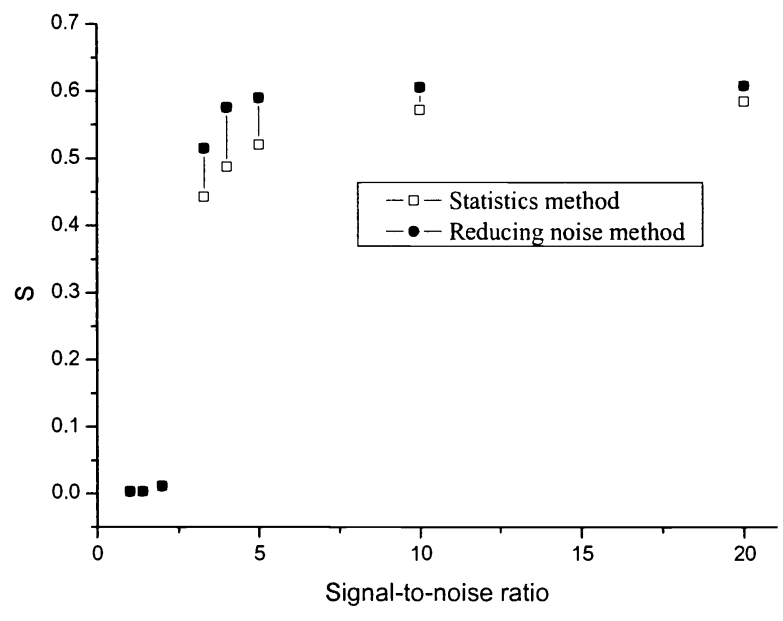

Fig. 9-1 Results of the computational results of numerical simulation by using the method for reducing noise effect.

(The pixel number for each subaperture is $2 \times 2$ )

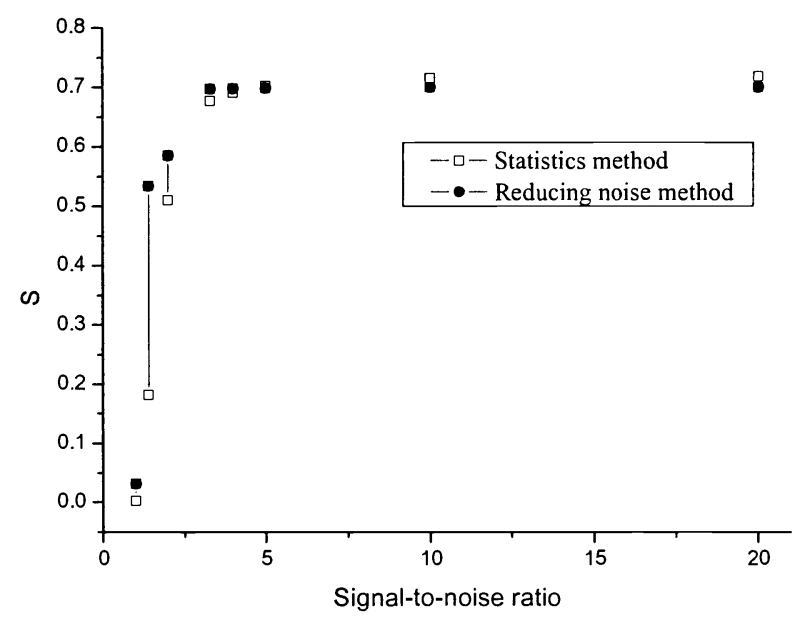

Fig. 9-2 Results of the computational results of numerical simulation by using the method for reducing noise effect.

(The pixel number for each subaperture is $4 \times 4$ )

Computational conditions: proportion of the deadspace is $0.125, \sigma_{r}=2$, others are same as those in Fig. 2 besides those indicated in this figure.

\section{CONCLUSIONS}

Based on our previous numerical simulation of effects of noise and detection error and numerical simulation of a dynamic AO system, modeling and a numerical simulation of the effects of noise and detection error in a dynamic AO system are realized in this paper. Numerical simulation investigations in a dynamic AO system show that (i) when the sampling density is larger than a certain value, the computational results are substantially same. This certain value can be thought as the necessary pixel number for the $\mathrm{AO}$ system on the corresponding condition. (ii) The size of focal spot should be large enough. When the size is suitable, the good result can be gotten. (iii) On the condition of practical deadspace proportion, the effect of deadspace is not important. But, when the pixel number for each subaperture is $2 \times 2$, we must reduce deadspace as we can. (iv) SNR must be larger than a certain value. Under the condition of smaller SNR than this value, the measurement error in the wavefront sensor increases rapidly to become unacceptable. (v) A statistics method is presented to evaluate the effects of readout noise and/or photon noise in a numerical simulation. On the condition of larger SNR, there is no significant difference between results by using the formulation method and by using the statistics method. However, as SNR gets smaller, the formulation method becomes less accurate. (vi) A simple method for reducing the noise effect is presented and preliminary computational results are presented as well. When the pixel number for each subaperture is $2 \times 2$, this method can improve the performance of AO system. It is shown that a numerical simulation is very valuable in design, examination, investigation and application of a highly complex electro-optic system such as an $\mathrm{AO}$ system. 


\section{ACKNOWLEDGEMENT}

The authors gratefully acknowledge the many helpful discussions with Prof. Genrui Cao of the Beijing Institute of Technology.

\section{REFERENCES}

1. R.K.Tyson, Principles of Adaptive Optics, 2nd Ed., Academic Press, Boston, 1997.

2. M.C.Roggeman, B.Welsh, Imaging Through Turbulence, CRC Press, Boca Raton, Florida, 1996.

3. R.V.Digumarthi, N.G.Mehta, R.M.Blankinship, "Effects of a realistic adaptive optics system on the atmospheric propagation of a high energy laser beam," Proc. SPIE Vol. 1221, pp.157-165, 1990.

4. C.A.Primmerman, T.R.Price, R.A.Humphreys, B.G.Zollars, H.T.Barclay, J.Hermann, “Atmospheric-compensation experiments in strong-scintillation conditions," Appl. Opt. 34, pp. 2081-2088, 1995.

5. S.N.Gullapalli, R.Abreu, W.M.Rappoport, W.P.Zmek, R.Pringle, "Modeling of the SAAO adaptive optics system," Proc. SPIE 3931, pp.285-299, 2000.

6. Hai-Xing Yan, Shu-Shan Li, She Chen, "Numerical simulation investigations of the dynamic control process and frequency response characteristics in an adaptive optics system," Proc. SPIE Vol. 4494, pp.156-166, 2002.

7. She Chen, Hia-Xing Yan, Shu-Shan Li, "Numerical simulation of an adaptive optics system: Effect of noise and detection error," Acta Optica Sinica 21 (5), pp.545-551, 2001. (in Chinese)

8. Hai-Xing Yan, She Chen, Shu-Shan Li, "Numerical simulation investigations of the effects of noise and detection error in an adaptive optics system," Proc. SPIE Vol. 4494, pp.144-155, 2002.

9. Genrui Cao, Xin Yu, “Accuracy analysis of Hartmann-Shack wavefront sensor operated with a faint object," Opt. Eng. 33, pp.2331-2335, 1994.

10. T.J.Kane, B.M.Welsh, C.S.Gardner, “Wavefront detector optimization for laser guided adaptive telescopes,” Proc. SPIE Vol. 1114, pp. 160-171, 1989.

11. Hai-Xing Yan, Shu-Shan Li, De-Liang Zhang, She Chen, "Numerical simulation of an adaptive optics system with laser propagation in the atmosphere," Appl. Opt. 39, pp.3023-3031, 2000.

12. Hai-Xing Yan, She Chen, De-Liang Zhang, Shu-Shan Li, "Numerical simulation of an adaptive optics system by means of modal wavefront reconstruction," Acta Optica Sinica 18 (1), pp.103-108, 1998. (in Chinese)

13. J.S.Morgan, D.C.Slater, J.G.Timothy, E.B.Jenkins, "Centroid position measurements and subpixel sensitivity variations with the MAMA detector,” Appl. Opt. 28, pp.1178-1192, 1989. 